Received: 25 July 2018

Accepted: 30 January 2019

Published online: 05 March 2019

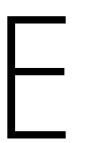

C
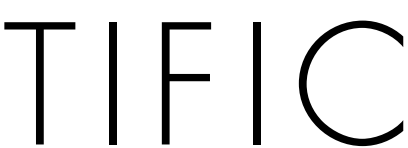

REP

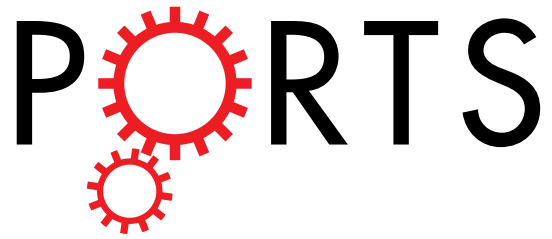

\title{
OPEN
}

\section{Relationship between Daily and In-laboratory Gait Speed among Healthy Community-dwelling Older Adults}

\author{
Naoto Takayanagi ${ }^{1}{ }^{1}$, Motoki Sudo $^{1}$, Yukari Yamashiro ${ }^{1}$, Sangyoon Lee ${ }^{2}$, \\ Yoshiyuki Kobayashi ${ }^{3}$, Yoshifumi Niki ${ }^{1}$ \& Hiroyuki Shimada $\mathbb{1}^{2}$
}

Gait speed in laboratory settings (in-laboratory gait speed) is one of the important indicators associated with the decline in functional abilities in older adulthood. Recently, it has become possible to measure gait speed during daily living (daily gait speed) using accelerometers. However, the relationship between these two gait speed parameters is unclear. This study aimed to compare in-laboratory gait speed, measured by a sheet-type pressure sensor, and daily gait speed, measured by an accelerometer, in healthy community-dwelling older adults. Participants were aged $\geq 60$ years, residing in Takahama city, Aichi, Japan. To calculate daily gait speed, participants were instructed to wear a tri-axial accelerometer on their waist. A total of 1965 participants were included in the final analysis. The results showed a weak association $(r=0.333, p<0.001)$ between the two gait speed parameters. Furthermore, average daily gait speed was significantly lower than average in-laboratory gait speed. However, both gait speed parameters declined significantly with age. These results suggest that, in addition to inlaboratory gait speed, daily gait speed may be a helpful parameter for predicting decline in functional abilities.

Gait speed is one of the important indicators of the decline in an individual's functional abilities, especially in older adults ${ }^{1-3}$. Previous studies reported that slow gait speed is associated with a loss of maintenance of instrumental activities of daily living (IADL) $)^{2}$, mild cognitive impairment (MCI $)^{4}$, and the risk of cardiovascular death ${ }^{5}$. Therefore, gait speed assessment in older adults can help predict the decline in their functional abilities.

Traditionally, gait speed has been measured and assessed mainly in laboratory settings by using a stopwatch, a tape measure, and a sheet-type pressure sensor ${ }^{6,7}$. Recently, it has become possible to assess gait speed in daily living (in free-living conditions) by using wearable sensors. Multiple researchers have assessed daily gait speed using a tri-axial accelerometer ${ }^{8-11}$

Although various studies have reported the methodology for and accuracy of gait speed measurements using wearable sensors, the relationship between daily gait speed and that in laboratory settings (in-laboratory gait speed) remains unclear. Previous studies reported that both gait speed parameters declined with age $e^{12,13}$. However, these two gait speed parameters were measured in different participants. Therefore, to clarify the relationship between the two gait speed parameters, it is essential to measure these within the same participants.

Accordingly, the present study aimed to measure daily gait speed using a tri-axial accelerometer, and in-laboratory gait speed using a sheet-type pressure sensor, among healthy community-dwelling older adults. This was done to confirm if these parameters decline with age. Another purpose was to compare between these parameters within the same participants. Daily gait speed has been measured continuously during gait in free-living conditions. However, because in-laboratory gait speed has only been measured several times in one measurement day, some participants may walk faster or more carefully during such assessments than in free-living conditions.

\footnotetext{
${ }^{1}$ Tokyo Research Laboratories, Kao Corporation, 2-1-3 Bunka, Sumida-ku, Tokyo, 131-8501, Japan. ${ }^{2}$ Department of Preventive Gerontology, Center for Gerontology and Social Science, National Center for Geriatrics and Gerontology, 7-430 Morioka, Obu, Aichi, 474-8511, Japan. ${ }^{3}$ Exercise motivation and Physical function Augmentation Research Team, Human Augmentation Research Center, National Institute of Advanced Industrial Science and Technology, Waterfront 3F, 2-3-26, Aomi, Koto-ku, Tokyo, 135-0064, Japan. Correspondence and requests for materials should be addressed to N.T. (email: takayanagi.naoto@kao.com)
} 


\begin{tabular}{|l|l|l|l|l|l|}
\hline & All $(\mathbf{n}=\mathbf{1 9 6 5})$ & $\begin{array}{l}\mathbf{6 0 - 6 9} \text { years } \\
(\mathbf{n}=\mathbf{1 0 1 3})\end{array}$ & $\begin{array}{l}\mathbf{7 0 - 7 9} \text { years } \\
(\mathbf{n}=775)\end{array}$ & $\begin{array}{l}\geq \mathbf{8 0} \text { years } \\
\mathbf{( n}=177)\end{array}$ & Significant difference \\
\hline Age (years) & $70.3 \pm 6.3$ & $65.2 \pm 2.6$ & $74.1 \pm 2.7$ & $82.6 \pm 2.6$ & $* p<0.001 \eta^{2}=0.826$ \\
\hline Sex (males: females) & $\begin{array}{l}766: 1199[39 \%: \\
61 \%]\end{array}$ & $396: 617[39 \%: 61 \%]$ & $304: 471[39 \%: 61 \%]$ & $66: 111[37 \%: 63 \%]$ & $\begin{array}{l}\chi^{2}=0.238 \\
p=0.888 V=0.011\end{array}$ \\
\hline Height $(\mathrm{cm})$ & $156.3 \pm 8.5$ & $158.0 \pm 8.2$ & $155.1 \pm 8.4$ & $151.5 \pm 8.5$ & $* p<0.001 \eta^{2}=0.055$ \\
\hline Weight $(\mathrm{cm})$ & $57.4 \pm 10.2$ & $58.9 \pm 10.7$ & $56.5 \pm 9.4$ & $52.4 \pm 8.9$ & ${ }^{*} p<0.001 \eta^{2}=0.035$ \\
\hline BMI $\left(\mathrm{kg} / \mathrm{m}^{2}\right)$ & $23.4 \pm 3.2$ & $23.5 \pm 3.3$ & $23.4 \pm 3.0$ & $22.8 \pm 3.1$ & $p=0.021 \eta^{2}=0.004$ \\
\hline Average steps (steps/day) & $6478.7 \pm 3021.3$ & $7062.5 \pm 2987.3$ & $6183.6 \pm 2947.9$ & $4429.8 \pm 2402.1$ & $* p<0.001 \eta^{2}=0.064$ \\
\hline
\end{tabular}

Table 1. Demographics of participants and average steps for each age group. Data are shown as the mean \pm SD. One-way ANOVAs or $\chi^{2}$ tests were conducted to compare age groups. $* p<0.05, \eta^{2}>0.01$.

\begin{tabular}{|c|c|c|c|c|c|c|c|}
\hline & \multirow{2}{*}{$\begin{array}{l}\text { All } \\
(n=1965)\end{array}$} & \multirow{2}{*}{$\begin{array}{l}\text { 60-69 years } \\
(n=1013)\end{array}$} & \multirow{2}{*}{$\begin{array}{l}70-79 \text { years } \\
(n=775)\end{array}$} & \multirow{2}{*}{$\begin{array}{l}\geq 80 \text { years } \\
(\mathrm{n}=177)\end{array}$} & \multicolumn{2}{|c|}{ Main effects } & \multirow{2}{*}{$\begin{array}{l}\text { Interaction effect } \\
\text { Age } \times \text { Measurement }\end{array}$} \\
\hline & & & & & Age & Measurement & \\
\hline $6.4-\mathrm{m}$ gait speed $(\mathrm{cm} / \mathrm{s})$ & $117.0 \pm 19.9$ & $122.3 \pm 18.3$ & $114.0 \pm 19.2$ & $100.6 \pm 20.9$ & \multirow{2}{*}{$\begin{array}{c}* p<0.001 \\
\eta^{2}=0.135\end{array}$} & \multirow{2}{*}{$\begin{array}{l}* p<0.001 \\
\eta^{2}=0.042\end{array}$} & \multirow{2}{*}{$p=0.260 \eta^{2}<0.001$} \\
\hline Daily gait speed $(\mathrm{cm} / \mathrm{s})$ & $109.7 \pm 22.6$ & $115.2 \pm 23.0$ & $105.8 \pm 21.2$ & $95.6 \pm 14.9$ & & & \\
\hline
\end{tabular}

Table 2. Comparison of 6.4-m gait speed and daily gait speed between age groups. Data are shown as the mean $\pm \mathrm{SD}$. Two-way repeated-measures ANOVAs were conducted. $* p<0.05, \eta^{2}>0.01$.

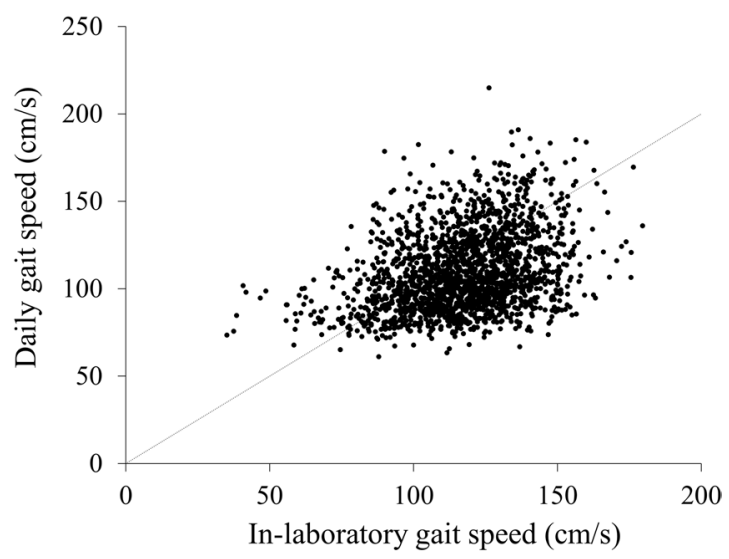

Figure 1. Relationship between daily and in-laboratory gait speed. The Pearson's correlation analysis was used to determine the relationship between the two gait speed parameters. $r=0.333, p<0.001 ; \mathrm{N}=1965$.

Therefore, we hypothesized that these two gait speed parameters would be inconsistent, because although participants may not be able to change their daily gait speed, they may be able to change their in-laboratory gait speed intentionally.

\section{Results}

Table 1 shows the demographic results and average step counts of participants in the different age groups. Significant differences between the age groups were found for age $\left(p<0.001, \eta^{2}=0.826\right)$, height $(p<0.001$, $\left.\eta^{2}=0.055\right)$, weight $\left(p<0.001, \eta^{2}=0.035\right)$, and average steps per day $\left(p<0.001, \eta^{2}=0.064\right)$.

Table 2 compares the two gait speed parameters between age groups and measurement methods. Significant main group effects were found for age group $\left(p<0.001, \eta^{2}=0.135\right)$ and measurement method $(p<0.001$, $\left.\eta^{2}=0.042\right)$. However, no interaction effect was found $\left(p=0.260, \eta^{2}<0.001\right)$.

Figure 1 shows the relationship between daily and in-laboratory gait speed. These two gait speed parameters were positively correlated $(r=0.333, p<0.001)$.

\section{Discussion}

This study aimed to compare daily gait speed, measured by tri-axial accelerometers, and in-laboratory gait speed among healthy community-dwelling older adults. We hypothesized that these two gait speed parameters would be inconsistent, because although participants may not be able to change their daily gait speed, they may be able to change their in-laboratory gait speed intentionally. Findings revealed only a weak association between daily and in-laboratory gait speed $(r=0.333, p<0.001)$. Additionally, the average daily gait speed was significantly lower than the average in-laboratory gait speed. These results support our hypothesis that the two gait speed parameters are inconsistent. 
A

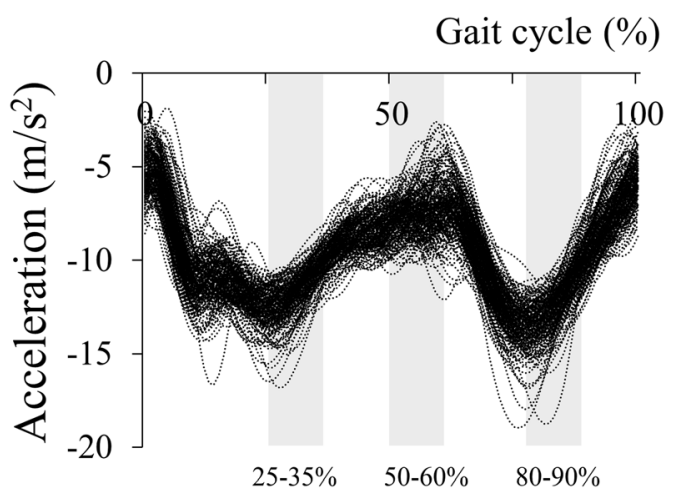

B
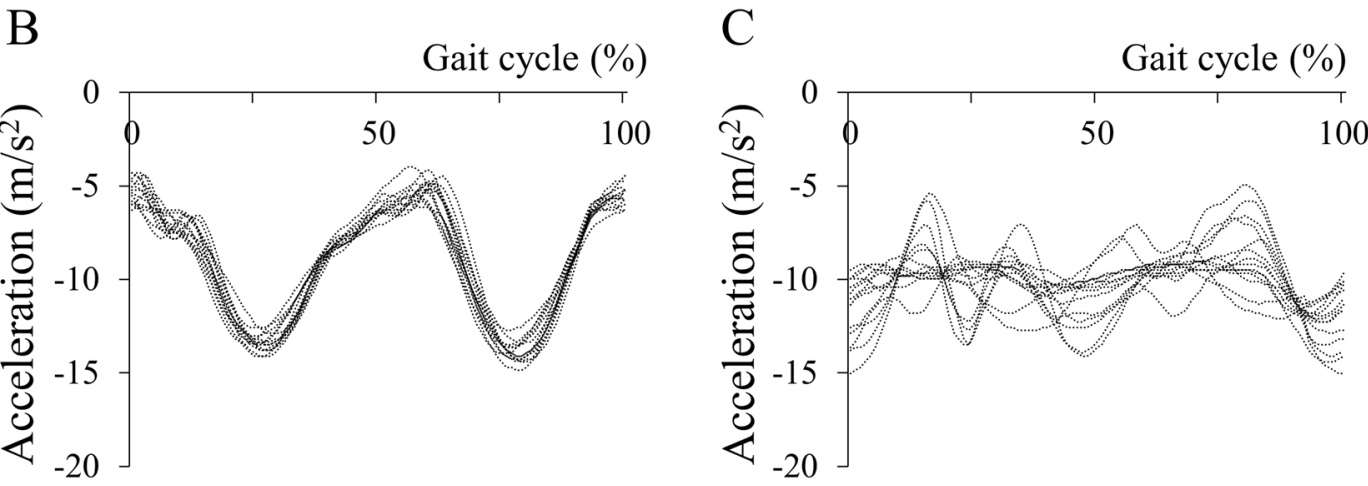

Figure 2. Accelerometer data from the Advanced Industrial Science and Technology (AIST) gait database and from when the device was correctly or incorrectly attached at the waist of the participants. (A) Vertical acceleration data for the right waist (anterior superior iliac spine) of 204 participants aged 20 to 77 from the AIST gait database ${ }^{28}$. (B) Example data for one participant with the accelerometer attached at the waist for all 14 days. (C) Data from another participant with no valid measurement days during the 14-day period. Based on the AIST gait database, if the average acceleration during $25-35 \%$ and $80-90 \%$ of the gait cycle is greater than that during $50-60 \%$ of the gait cycle, the acceleration data are excluded from analysis.

In the present study, we measured daily gait speed across 14 days, and the criteria for inclusion in the analysis were wearing the accelerometer for a total duration of $\geq 7$ days, for $\geq 10$ hours/day. This standard was used in previous studies ${ }^{14,15}$ to measure the total amount of activity in a day. This enabled us to obtain a daily gait speed measure which is not affected by the time and the day. On the other hand, in-laboratory gait speed was measured on one measurement day, where it may be easier for subjects to change their gait speed intentionally. This may explain the lack of a strong association between these parameters in the present study.

The present findings also showed that the average daily gait speed was significantly lower than that in the laboratory. Multi-tasking during walking in daily living conditions could explain this result. A previous study on older adults reported that the average gait speed in laboratory settings with dual tasks (a cognitive task) was significantly slower than that with no task ${ }^{16}$. In daily living, people sometimes walk while performing other tasks. This may be one of the reasons why daily gait speed was significantly lower than in-laboratory gait speed.

Another previous study reported that the usual gait speed is affected by psychological factors ${ }^{17}$. This study showed that the 'body pain' and 'vitality' scores in the 12-item Short-Form Health Survey (SF-12; Health Related Quality of Life scale) were independent predictors of six-metre gait speed (i.e. in-laboratory gait speed) in older adults ${ }^{17}$. In particular, the body pain score changes depending on the individual's condition on that day. Some participants may walk faster than usual while enduring the pain in laboratory settings. On the other hand, it may be difficult for participants with body pain to walk faster than usual at all times in daily living. Future studies need to clarify the association between daily gait speed and these factors.

Although a significant difference was observed between the two gait speed parameters, the present study showed that both these parameters declined with age. Multiple previous studies have reported a significant decline in in-laboratory gait speed with age ${ }^{12,18,19}$. However, for daily gait speed, Schimpl et al. ${ }^{13}$ only reported the measurement of daily gait speed in healthy participants aged 17-65 years, finding a slight decline of this parameter with increasing age. In the present study, the daily gait speed of 1965 healthy community-dwelling older adults aged over 60 years was measured and a decline with age, similar to that found for in-laboratory gait speed, was observed. These results suggest that, as for in-laboratory gait speed, daily gait speed may be a parameter associated with the decline in functional abilities in older adults. This finding may provide a good basis for the assessment of functional decline in free-living conditions. 
Many previous studies reported that in-laboratory gait speed is associated with the decline in an individual's functional abilities ${ }^{1-4}$. Cesari et al. reported that a usual gait speed in laboratory settings of less than $100 \mathrm{~cm} / \mathrm{s}$ indicates a high risk of negative health-related outcomes in well-functioning older adults ${ }^{20}$. However, while the number of participants whose in-laboratory gait speed was less than $100 \mathrm{~cm} / \mathrm{s}$ was 381 (19.4\%), that of participants with a daily gait speed of less than $100 \mathrm{~cm} / \mathrm{s}$ was $779(39.6 \%)$. These points suggest that standard expected values or values indicating high-risk individuals need to be determined separately for daily and in-laboratory gait speed, to predict decline in functional abilities more accurately. Future studies on the relationships between daily gait speed and individuals' functional decline are needed.

Because daily gait speed has been measured by the accelerometer in daily living, this gait parameter can be assessed frequently. In comparison, it is difficult to measure in-laboratory gait speed continuously. High frequency measurements enable the detection of changes in parameters at earlier stages. Therefore, the continuous measurement of daily gait speed may be useful to detect the decline in the functional abilities of older adults.

The present study focused on daily gait speed. Recently, some other gait parameters derived from walking acceleration signal have also been assessed. For instance, Ho et al. proposed a method for step length estimation at various gait speeds by using a smartphone ${ }^{21}$. Additionally, Manor et al. suggested a smartphone-based assessment of stride time during gait in non-laboratory settings ${ }^{22}$. These gait parameters have been associated with aging ${ }^{23,24}$. Therefore, it may be possible to detect the decline in functional abilities more accurately by using daily gait speed and these parameters together.

There are several limitations in this study. First, the type of shoes worn during gait measurements were not considered. Second, the accelerometer has an LCD screen and participants could see their step counts. Therefore, the amount of activity during the assessment period might have been higher relative to that on ordinary free-living days ${ }^{25}$.

In the present study, we compared daily gait speed, measured by tri-axial accelerometers, and in-laboratory gait speed, measured by a sheet-type pressure sensor, in healthy community-dwelling older adults. The results showed only a weak association between daily and in-laboratory gait speed. Additionally, daily gait speed was significantly lower than that observed in the laboratory. However, both these parameters declined with age. These results suggest that daily gait speed may be a useful parameter for the prediction of functional decline among healthy community-dwelling older adults in free-living conditions.

\section{Methods}

Participants. The present study was based on data from the Takahama Study of Health Promotion for Older Adults conducted from September 2015 to June 2016. This study is a part of the National Center for Geriatrics and Gerontology Study of Geriatric Syndromes (NCGG-SGS), which is a cohort study that aimed to establish a screening system for geriatric syndromes ${ }^{26}$. The inclusion criteria for this study were age $\geq 60$ years and residence in Takahama city, Aichi, Japan. A total of 4072 community-dwelling older adults participated and agreed to wear the accelerometer during this study. All participants provided written informed consent by reading and signing a consent form that was approved by the institutional review board. This study was carried out in accordance with the guidelines proposed in the Declaration of Helsinki, and the study protocol was approved by the research ethics committee of the National Center for Geriatrics and Gerontology (Approval Number 861).

Daily data collection and analysis. The participants were instructed to wear the tri-axial accelerometer (HW-100, Kao Corporation, Tokyo, Japan) on their waist at all times while awake, except during swimming or bathing, and to maintain their usual activities. Furthermore, they were instructed to visit one of 75 designated places named as KENKOJISEICHI ${ }^{14}$ in Takahama city, once in 30-40 days, where the data measured by the accelerometer were downloaded onto a tablet computer using a near field communication (NFC) system (RC-380, Sony Corporation, Tokyo, Japan). KENKOJISEICHI included public facilities, gyms, drug stores, cafeterias, and beauty salons, in order to make it as easy as possible for the participants to visit a location and have their data downloaded.

Individuals were excluded if: (i) they did not visit KENKOJISEICHI within 60 days of the date of instruction to begin wearing the accelerometer $(n=1148)$; (ii) in-laboratory gait speed measurement was not conducted within the Takahama Study of Health Promotion for Older Adults $(\mathrm{n}=1)$; and (iii) participants were unable to meet the criteria for analysis of accelerometer data $(n=958)$. The criteria for analysis were wearing the accelerometer on their waist for a total duration of $\geq 7$ days, for $\geq 10$ hours/day, during the first 14 days after the day they began wearing the accelerometer. The remaining 1965 participants (48.3\%) were included in the final analysis.

Daily gait speed measurement. Daily gait speed was measured using an accelerometer (HW-100) for continuous monitoring during daily living. HW-100 is a tri-axial accelerometer providing 40 days of continuous recording at a sampling frequency of $64 \mathrm{~Hz}^{14}$. This device detects step cycle during gait ranging from 70 to 160 steps/min by medio-lateral and vertical acceleration. It commences recording of tri-axial acceleration during the gait cycle if the measured acceleration of the current cycle and the preceding two cycles are within $10 \%$ of one another. Therefore, the accelerometer records tri-axial acceleration during steady-state periods of gait. The generic structure of a gait speed estimation method is as follows: (1) the accelerometer is attached to the body and measures the acceleration signals; (2) with these measurements, mathematical models can be used to estimate gait speed $^{27}$. In the case of the HW-100, daily gait speed was calculated with a model that used composite acceleration during one gait cycle from the tri-axial acceleration measurements. The estimation accuracy of gait speed for this device is shown in Supplementary Fig. S1. Based on the accuracy evaluation conducted in the present study, as evident from Supplementary Fig. S1, the systematic error for this device was $14.3 \mathrm{~cm} / \mathrm{s}$, which suggests that this device may have underestimated daily gait speed as compared with the gold standard. Therefore, in the present 
study daily gait speed was adjusted by adding $14.3 \mathrm{~cm} / \mathrm{s}$ to the observed value. An average daily gait speed for valid days during a 14-day period was obtained for each participant.

The HW-100 accelerometer also measured step counts and wearing time ${ }^{14}$. For inclusion in the present study, a valid day was defined as a day on which the accelerometer was worn for $\geq 10$ hours $^{14,15}$. Data from the Advanced Industrial Science and Technology (AIST) gait database ${ }^{28}$ were used to define days on which the accelerometer was not correctly worn on the waist, to enable exclusion from analysis. This procedure is illustrated in Fig. 2, which shows the vertical acceleration data from 204 individuals in the AIST gait database and examples of data from present participants who wore the accelerometer correctly and incorrectly.

In-laboratory gait speed measurement. In the present study, 6.4-m gait speed was defined as in-laboratory gait speed based on a previous study ${ }^{29}$. This parameter was measured using a sheet-type pressure sensor (2.4-m long; Walk Way, Anima Corporation, Tokyo, Japan) placed in the middle of a 6.4-m walkway, operating at a sampling frequency of $100 \mathrm{~Hz}^{7}$. The participants were instructed to walk along the walkway at a comfortable pace. They repeated the $6.4-\mathrm{m}$ walk five times and the average in-laboratory gait speed was calculated.

Statistics. One-way measures analysis of variance (ANOVA) and the $\chi^{2}$ test were conducted to analyse physical characteristics and average steps per day between different age groups (60-69 years, 70-79 years, 80 years and over). A two-way repeated measures ANOVA was used to assess the main and interaction effects between age groups (60-69 years, 70-79 years, 80 years and over), and gait speed parameters (daily and in-laboratory gait speed). Furthermore, the relationship between the two gait speed parameters was examined by calculating Pearson's correlation coefficients $(r)$. The differences in the means were considered statistically significant if the $p$ values were less than 0.05 , the partial $\eta^{2}$ values were greater than 0.01 , and the $V$ values were greater than $0.10^{30}$. All statistical analyses were performed using the SPSS statistical software package (IBM SPSS Statistics Version 23, SPSS Inc., Chicago, IL, USA).

\section{Data Availability}

The datasets generated and/or analysed during the current study are not publicly available due to intellectual property reasons, but are available on reasonable request.

\section{References}

1. Shinkai, S. et al. Walking speed as a good predictor for the onset of functional dependence in a Japanese rural community population. Age Ageing 29, 441-446, https://doi.org/10.1093/ageing/29.5.441 (2000).

2. Suzuki, T. et al. Walking speed as a good predictor for maintenance of I-ADL among the rural community elderly in Japan: A 5-year follow-up study from TMIG-LISA. Geriatr. Gerontol. Int. 3, S6-S14, https://doi.org/10.1111/j.1444-0594.2003.00090.x (2003).

3. Tainaka, K., Takizawa, T., Katamoto, S. \& Aoki, J. Six-year prospective study of physical fitness and incidence of disability among community-dwelling Japanese elderly women. Geriatr. Gerontol. Int. 9, 21-28, https://doi.org/10.1111/j.1447-0594.2008.00492.x (2009).

4. Buracchio, T., Dodge, H. H., Howieson, D., Wasserman, D. \& Kaye, J. The trajectory of gait speed preceding mild cognitive impairment. Arch. Neurol. 67, 980-986, https://doi.org/10.1001/archneurol.2010.159 (2010).

5. Dumurgier, J. et al. Slow walking speed and cardiovascular death in well functioning older adults: prospective cohort study. BMJ 339, b4460 (2009).

6. Youdas, J. W. et al. Agreement between the GAITRite walkway system and a stopwatch-footfall count method for measurement of temporal and spatial gait parameters. Arch. Phys. Med. Rehabil. 87, 1648-1652, https://doi.org/10.1016/j.apmr.2006.09.012 (2006).

7. Makino, K. et al. Fear of falling and gait parameters in older adults with and without fall history. Geriatr. Gerontol. Int. 17, 2455-2459, https://doi.org/10.1111/ggi.13102 (2017).

8. Zijlstra, W. \& Hof, A. L. Assessment of spatio-temporal gait parameters from trunk accelerations during human walking. Gait Posture 18, 1-10 (2003).

9. Song, Y., Shin, S., Kim, S., Lee, D. \& Lee, K. H. Speed estimation from a tri-axial accelerometer using neural networks. Conf. Proc. IEEE Eng. Med. Biol. Soc. 3224-3227, https://doi.org/10.1109/IEMBS.2007.4353016 (2007).

10. Schimpl, M., Lederer, C. \& Daumer, M. Development and validation of a new method to measure walking speed in free-living environments using the actibelt ${ }^{\circledR}$ platform. PLoS One 6, e23080, https://doi.org/10.1371/journal.pone.0023080 (2011).

11. Zihajehzadeh, S. \& Park, E. J. Regression model-based walking speed estimation using wrist-worn inertial sensor. PLoS One 11, e0165211, https://doi.org/10.1371/journal.pone.0165211 (2016).

12. Himann, J. E., Cunningham, D. A., Rechnitzer, P. A. \& Paterson, D. H. Age-related changes in speed of walking. Med. Sci. Sports Exerc. 20, 161-166 (1988).

13. Schimpl, M. et al. Association between walking speed and age in healthy, free-living individuals using mobile accelerometry-a crosssectional study. PLoS One 6, e23299, https://doi.org/10.1371/journal.pone.0023299 (2011).

14. Jung, S. et al. Effectiveness of the KENKOJISEICHI local revitalization system on cognitive function change in older adults with mild cognitive impairment: study protocol for a randomized controlled trial. Trials 19, 276, https://doi.org/10.1186/s13063-018-2642-3 (2018).

15. Jung, S. et al. Relationship between physical activity levels and depressive symptoms in community-dwelling older Japanese adults. Geriatr. Gerontol. Int. 18, 421-427, https://doi.org/10.1111/ggi.13195 (2018).

16. Yogev-Seligmann, G. et al. How does explicit prioritization alter walking during dual-task performance? Effects of age and sex on gait speed and variability. Phys. Ther. 90, 177-186, https://doi.org/10.2522/ptj.20090043 (2010).

17. Tiedemann, A., Sherrington, C. \& Lord, S. R. Physiological and psychological predictors of walking speed in older communitydwelling people. Gerontol. 51, 390-395, https://doi.org/10.1159/000088703 (2005).

18. Samson, M. M. et al. Differences in gait parameters at a preferred walking speed in healthy subjects due to age, height and body weight. Aging (Milano) 13, 16-21 (2001).

19. Murray, M. P., Kory, R. C. \& Clarkson, B. H. Walking patterns in healthy old men. J Gerontol. 24, 169-178 (1969).

20. Cesari, M. et al. Prognostic value of usual gait speed in well-functioning older people-results from the Health, Aging and Body Composition Study. J. Am. Geriatr. Soc. 53, 1675-1680, https://doi.org/10.1111/j.1532-5415.2005.53501.x (2005).

21. Ho, N. H., Truong, P. H. \& Jeong, G. M. Step-detection and adaptive step-length estimation for pedestrian dead-reckoning at various walking speeds using a smartphone. Sensors (Basel) 16, 1423, https://doi.org/10.3390/s16091423 (2016).

22. Manor, B. et al. Smartphone app-based assessment of gait during normal and dual-task walking: demonstration of validity and reliability. JMIR Mhealth Uhealth 6, e36, https://doi.org/10.2196/mhealth.8815 (2018). 
23. Judge, J. O., Davis, R. B. \& Ounpuu, S. Step length reductions in advanced age: the role of ankle and hip kinetics. J. Gerontol. A. Biol. Sci. Med. Sci. 51, M303-312, https://doi.org/10.1093/gerona/51A.6.M303 (1996).

24. Hausdorff, J. M., Rios, D. A. \& Edelberg, H. K. Gait variability and fall risk in community-living older adults: a 1-year prospective study. Arch. Phys. Med. Rehabil. 82, 1050-1056, https://doi.org/10.1053/apmr.2001.24893 (2001).

25. Bravata, D. M. et al. Using pedometers to increase physical activity and improve health: a systematic review. JAMA 298, 2296-2304, https://doi.org/10.1001/jama.298.19.2296 (2007).

26. Shimada, H. et al. Driving continuity in cognitively impaired older drivers. Geriatr. Gerontol. Int. 16, 508-514, https://doi. org/10.1111/ggi.12504 (2016).

27. Yang, S. \& Li, Q. Inertial sensor-based methods in walking speed estimation: a systematic review. Sensors (Basel) 12, 6102-6116, https://doi.org/10.3390/s120506102 (2012).

28. Kobayashi, Y., Hobara, H. \& Mochimaru, M. AIST Gait Database 2015, https://www.dh.aist.go.jp/database/gait2015/index.html (2015).

29. Makizako, H. et al. Cognitive functioning and walking speed in older adults as predictors of limitations in self-reported instrumental activity of daily living: prospective findings from the Obu Study of Health Promotion for the Elderly. Int. J. Environ. Res. Public Health 12, 3002-3013, https://doi.org/10.3390/ijerph120303002 (2015).

30. Cohen, J. Statistical power analysis for the behavioral sciences (2nd ed.) 273-406 (Lawrence Erlbaum Associates, 1988).

\section{Acknowledgements}

We thank the research and healthcare staff involved in this study for their assistance with the assessments: Sungchul Lee, Seongryu Bae, Songee Jung, Keitaro Makino, Ippei Chiba, Kazuhiro Harada, and Kenji Harada, members of National Center for Geriatrics and Gerontology, and Kazushi Kato, Kazushi Isomura, Satomi Higashi, Sakura Nagata, Aya Iwasaki, and Yoshie Okada, members of the Health Promotion Group in Takahama city. We also thank Masataka Sasaki and Tatsuhiro Hara at ACOS Corporation, for their help with the data analysis. This work was supported by a Research Funding for Longevity Sciences (grant number: 27-22) from the National Center for Geriatrics and Gerontology, Grant-in-Aid for Scientific Research (A) (KAKENHI grant number: 26242059), and expenses for R\&D commissioned from Takahama city. This work was conducted in association with the Kao Corporation and the ALCARE Corporation. The sponsors of the study had no role in the study design, data collection and analysis, decision to publish, or preparation of the manuscript.

\section{Author Contributions}

Study conception and design: H.S. Data collection: N.T., M.S., Y.Y. and S.L. Data analysis and interpretation: N.T., M.S., Y.Y. and Y.N. Drafting manuscript: N.T. Critical revision: S.L., Y.K. and H.S. All authors read and approved the final manuscript.

\section{Additional Information}

Supplementary information accompanies this paper at https://doi.org/10.1038/s41598-019-39695-0.

Competing Interests: N.T., M.S., Y.Y. and Y.N. are employed by the Kao Corporation. Y.K. has received a research grant from the Kao Corporation. S.L. and H.S. have received research grants from the Kao Corporation and the ALCARE Corporation.

Publisher's note: Springer Nature remains neutral with regard to jurisdictional claims in published maps and institutional affiliations.

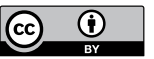

Open Access This article is licensed under a Creative Commons Attribution 4.0 International License, which permits use, sharing, adaptation, distribution and reproduction in any medium or format, as long as you give appropriate credit to the original author(s) and the source, provide a link to the Creative Commons license, and indicate if changes were made. The images or other third party material in this article are included in the article's Creative Commons license, unless indicated otherwise in a credit line to the material. If material is not included in the article's Creative Commons license and your intended use is not permitted by statutory regulation or exceeds the permitted use, you will need to obtain permission directly from the copyright holder. To view a copy of this license, visit http://creativecommons.org/licenses/by/4.0/.

(C) The Author(s) 2019 